\title{
La langue sous haute surveillance
}

\section{Anne-Françoise Allaz}

Prof. Dr méd., membre de la rédaction

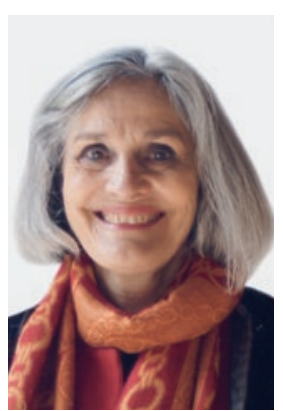

Les vifs débats concernant le langage "politiquement correct» envahissent depuis quelques temps les espaces médiatiques européens. Ils sont en lien avec le développement de la woke culture que revendiquent les militants awoken, c'est-à-dire «éveillés» aux discriminations raciales, de genre, de religion.

Parmi d'autres facettes de la défense des droits des «minorités», il y a la révision de certains aspects discriminatifs de la langue. Nous connaissons la féminisation de celle-ci par l'écriture inclusive ou la modification de dénominations à l'image de malentendant (au lieu de sourd), de personne handicapée (au lieu de handicapé), etc. Tous ces changements marquent un souhait de déstigmatisation ou de reconnaissance salutaire.

Les excès identitaires inspirés du mouvement woke ont vite été politisés. «Politiquement correct» est devenu un reproche, voire une insulte. Est-ce de la moralité ou de la censure? La survalorisation des minorités, le communautarisme sont dénoncés comme portant un risque de déconstruction des normes communes qui touchent à l'héritage des Lumières (Aufklärung). Pour beaucoup, heureusement, la protection des minorités ne s'érige ni contre la liberté d'expression, ni contre le partage de valeurs. Toujours est-il que la langue est devenue un enjeu sous haute surveillance. C'est que les mots ont du pouvoir! Relisons Michel Foucault: "La façon dont nous parlons du monde et la façon dont nous le vivons sont inextricablement liées.»

Trois exemples parmi tant d'autres montrent combien la médecine est concernée.

- Un neurologue hospitalier américain déplore dans le JAMA [1] la recommandation qui lui est faite d'éviter toute description raciale, ethnique ou du pays d'origine du patient dans les dossiers médicaux. Je me dis que l'anamnèse sociale est là sacrifiée à une norme "politiquement correcte», qui rétrécit de manière regrettable l'information médicalement utile. Mais un récent article du Lancet [2] me tance sévèrement: «il faut reconsidérer l'indication de la race/ ethnie dans les notes de consultation»; c'est du "racisme structurel dont souffre le système de santé». Je reste perplexe: «Patiente de 25 ans, réfugiée, d'origine éthiopienne», discriminatoire, péjoratif? Déci- dément, il me semble que non. L'indication de l'origine de la patiente référée informe sur sa situation médico-psycho-sociale et permet d'organiser, par exemple, un accueil comprenant un temps de consultation suffisant.

- Autre exemple: sous l'administration Trump, fin 2017, le Center for Disease Control (CDC) a reçu la demande d'éviter l'utilisation de sept mots dans les textes officiels et demandes de fonds, dont: «vulnérable», «diversité», "transgenre», «fœtus», «evidence et science-based» [3]. Le caractère fortement idéologique d'une telle directive n'échappera à personne, de même que ses conséquences sur les orientations de santé publique et de recherche. Cette tentative d'escamoter des mots rappelle la novlangue décrite par Georges Orwell dans son livre 1984, où l'on détruit les mots ne correspondant pas à la pensée unique: «Ne voyez-vous pas que le véritable but de la novlangue est de restreindre les limites de la pensée?»

- Enfin, il me semble clair que la novlangue technocratique avec son projet de contrôle pourrait augmenter son emprise, si nous ne restons pas vigilants. Elle nous assigne déjà le rôle de prestataires de soin à des clients, bien loin de la complexité de la relation médecin-malade.

Pour moi, l'espace de la médecine reste celui où tout disons presque tout - peut (encore) se dire et, s'il le faut, rester secret. En pratique, nous le savons, nous devons maintenir l'équilibre entre la liberté de parole et le respect d'autrui, tenter d'accueillir avec toute la tolérance et la bienveillance possibles la diversité et la vulnérabilité sous leurs différentes présentations. Cela fait partie du serment d'Hippocrate.

Pour ce faire, notre liberté d'expression, donc de pensée, est précieuse, comme notre personnalité et notre créativité. Ne nous en laissons dépouiller à aucun prix!

\section{Références}

1 Berger JR. Political Correctness of Medical Documentation. JAMA Neurology. 2015;72:624-5.

2 Cerdeña JP, et al. From race-based to race-conscious medicine: how anti-racist uprisings call us to act. Lancet. 2020;396:1125-8.

3 Castro K, et al. Seven Deadly Sins Resulting from the CDC's Seven Forbidden Words. Ann Intern Med. 2018;168:513-5. 